\title{
Prospective Physics Teachers' Consistency and Scientific Reasoning in the Learning of Force Concept
}

\author{
S. P. Sriyansyah \\ Department of Physics Education \\ Postgraduate School, Universitas Pendidikan Indonesia \\ Bandung, Indonesia \\ syakti.perdana.sriyansyah@student.upi.edu
}

\author{
D. Saepuzaman \\ Department of Physics Education \\ Universitas Pendidikan Indonesia \\ Bandung, Indonesia \\ dsaepuzaman@upi.edu
}

\begin{abstract}
Physics education research has been revealed that scientific reasoning was a hidden variable which influences student's conceptual understanding in physics. A student who has good understanding is characterized by their scientific consistency and representational consistency. Hence, this study was conducted to investigate particularly the relation between the prospective physics teacher's conceptual understanding, scientific consistency, representational consistency and scientific reasoning, as well as a depiction of each variable, in the learning of force concept. Representational Variant of Force Concept Inventory (R-FCI) was used to collect consistency data and Lawson Classroom Test of Scientific Reasoning was used for scientific reasoning data. The correlation between assessed variables was analized by using the Spearman rank correlation. The participant was 29 first-year prospective physics teachers who attended for introductory physics courses in 2015 at Indonesia University of Education. The findings showed that none prospective physics teacher is formal reasoner, while respectively $31 \%$ concrete reasoner, $45 \%$ early transitional reasoner and $24 \%$ late transitional reasoner. As for the scientific consistency and representational consistency, both of them can be classified as either moderately consistent or inconsistent, with none consistent. The correlation coefficient between prospective physics teacher's scientific reasoning and both consistency was 0.48 for scientific consistency and 0.39 for representational consistency, while the correlation between their scientific reasoning and conceptual understanding of the force concept was 0.49. Through this investigation we provide useful information to consider scientific reasoning which influences the student's conceptual understanding and both consistency in the learning of the force concept in the physics classroom at high or middle school.
\end{abstract}

Keywords-representational consistency; scientific consistency; scientific reasoning; force concept

\section{INTRODUCTION}

Conceptual understanding is the most concern in physics education research. A large body of research focuses on aspects of students' understanding, including the influencing factors. The researchers found among these factors are the scientific reasoning and representational consistency [1-5].

Scientific reasoning becomes a hidden variable that influences students' understanding [1]. Researchers showed a significant correlation between the single student normalized Force Concept Inventory (FCI) gain and students' scientific reasoning prescore at several universities in the U.S. [1-3].
Scientific reasoning then becomes important to identify at the beginning of the instruction, as well as with the representational consistency.

Nieminen reported that the representational consistency is positively correlated with students' improved understanding scores of the force concept [5]. Representational consistency is one indicator of a student already has a good understanding, while another indicator is scientific consistency. A student who has a good conceptual understanding can be marked on their ability to solve problems even though the context and the representation are different [6].

Previous research has indeed been reported that scientific reasoning [1-4] and representational consistency [5] are factors that affect students' understanding, but these reports have not provided a comprehensive explanation of how the relationship between the two factors. The existing report like Nieminens' report [5] is limited in that it only provides the correlation coefficient between the reasoning and representational consistency prescores associated with an explanation of the correlation of each factor with single student normalized FCI gain. It does not provide an explanation of how both factors correlate each other, as well as how scientific reasoning correlate with scientific consistency and students' understanding which measured using R-FCI. This deficiency led us to conduct an investigation, which aims to examine the relation between students' scientific reasoning and the representational consistency to confirm earlier findings [5], as well as providing a theoretical explanation of the relation between both assessed variables. We want to see if the results of this study also provide the same results with the previous study for the education setting in Indonesia.

Our second aim was to investigate the relations between the students' conceptual understanding which measured by using R-FCI and scientific reasoning, as well as also the relation between students' scientific consistency and scientific reasoning. We want to clarify whether or not the student conceptual understanding is correlated with scientific reasoning when the understanding measured using the R-FCI test to replace FCI test. As Nieminen argued that R-FCI has been representative and can be used to measure students' understanding of the concept of force [7]. In this study, we administer the test at the end of the instruction as well as to reveal the students' scientific reasoning and consistency 
profiles and how they correlate each other, not to reveal the extent to the which it evolved as an impact of learning pedagogy.

Representational consistency is defined as the ability to solve isomorphic problem, i.e. which has same context, but with different representations, consistently even though it is incorrect scientifically, while if solving isomorphic problem consistently and scientifically correct, it is called the scientific consistency. Both types of consistency relate to the ability to use multiple-representation, but though the ability to interpret multiple-representations is required, but it is not sufficient to understand the concept of physics correctly [7].

Students who have a good conceptual understanding will also demonstrate a good consistency. Constructing a good understanding requires the ability to establish logical relations between scientific facts and prior conception by using representations and this ability named as scientific reasoning [8]. Therefore, it is reasonable to say that scientific reasoning seems to have a correlation with consistency, either with scientific consistency or representational consistency.

Reasoning ability is very essential in scientific thinking. Lawson [9] defines the scientific reasoning ability as the ability to use the thinking patterns, such as combinatorial (F1), identification and control of variables (F2), proportional (F3), probabilistic (F4), correlational (F5), and hypotheticaldeductive (F6). Those thinking patterns included in the formal thinking patterns, while for concrete thinking patterns consist of class inclusion $(\mathrm{C} 1)$, conservation $(\mathrm{C} 2)$ and serial ordering (C3). The identification of the student reasoning pattern is very useful to know the students who experienced difficulty in understanding the concept and assess how effective of learning that has been done in class [3].

Scientific reasoning closely related to the intellectual development theory proposed by Piaget. Based on Piaget's theory, Lawson [10] divides the students into three categories thinkers, are concrete thinkers (empirical-inductive thinking), transitional thinker (towards formal), and formal thinker (hypothetical-deductive thinking). Students who are concrete thinkers have not been able to think proportional logically, so that such students should learn by interacting directly with the material being studied. Concrete thinker must struggle if they had a problem out of context and feel so difficulty with abstract concepts and hypothetical tasks. While students who are formal thinker had been able to think abstractly in the form, "if ..., then ..., so ...," or propositional logic, reasoning logically, draw conclusions from the information provided, and use the appropriate logic to the hypothetical situation in diverse contexts [11]. Finally, transitional thinkers only able to complete hypothetical tasks in some contexts

\section{RESEARCH METHOD}

This study investigated the variables include: scientific reasoning, representational consistency, scientific consistency and conceptual understanding. Participants involved are 29 prospective physics teacher student who took an introductory physics course in the second semester of the first year 2015. The scientific reasoning is measured using a modified version of the Lawson Classroom Test of Scientific Reasoning
(LCTSR) [12], while three other variables using Representational Variant of the Force Concept Inventory (RFCI) [7]. Both tests are given at the end of instruction after participants learned about the concept of force.

Consistency scores were analyzed by the rules set by Nieminen et. al. [7] and is divided into three categories, namely consistent, moderately consistent and inconsistent. Conceptual understanding scored by the binary scoring method, a score of 1 if the question is answered correctly and a score of 0 if any, for 27 R-FCI items. Based on LCTSR scores obtained, students are categorized into four categories reasoner, the concrete reasoner, early transitional reasoner, late transitional reasoner and formal reasoner.

All collected data were analyzed to determine the percentage of students in each category and the correlation between assessed variables. The statistics are used to calculate the correlation between variables is the Spearman rank correlation because of the variables involved are not normally distributed.

\section{RESULTS AND DISCUSSION}

Table 1 shows the percentage of prospective physics teacher student in each consistency and scientific reasoning category. None of the students who are in the category of formal reasoner and scientifically consistent. Only one person showed consistently in representational consistency. All of them can be classified as either concrete or transitional reasoner for scientific reasoning test and as either moderately consistent or inconsistent for scientific consistency test.

Comparison of the students percentage in each category for scientific and representational consistency suggests that even half the students demonstrated moderately consistent on representational consistency, but almost entirely inconsistent on scientific consistency. That means students have more difficulty in scientific concepts in question, not on the representation used. This indication was strengthened by the low average score of students' conceptual understanding, i.e. 17 out of 100 .

Four central concepts in the R-FCI is a theoretical content, abstract and conceptual knowledge. Difficulties encountered by students seem to be in line with the level of their intellectual development. Nearly one-third and half of the students is a concrete and early transitional thinker, respectively. Students who have concrete reasoning pattern characterized by:

(1) searches for and identifies some variables influencing a phenomenon, but does so unsystematically; (2) makes observations and draws inferences from them, but in causal contexts does not initiate reasoning with the hypothetical - the possible (i.e., does not use if/and/then reasoning to test causal hypotheses); (3) responds to difficult problems by applying a related but not necessarily correct rule; and (4) processes information but is not aware of his/her own reasoning (e.g., does not check his/her own conclusions against the given data or other experience; is not reflective; does not think about his/her thinking) [10, 13]. 
TABLE I. CATEGORY FOR CONSISTENCY AND SCIENTIFIC REASONING TESTS $(\mathrm{N}=29)$

\begin{tabular}{|c|l|c|}
\hline No. & \multicolumn{1}{|c|}{ Category } & Student proportion \\
\hline$\# \mathbf{1}$ & Representational consistency & \\
\hline & $\bullet$ Consistent & $3 \%$ \\
\hline & $\bullet$ Moderately consistent & $55 \%$ \\
\hline & $\bullet$ Inconsistent & $41 \%$ \\
\hline$\# \mathbf{2}$ & Scientific consistency & $0 \%$ \\
\hline & $\bullet$ Consistent & $3 \%$ \\
\hline & $\bullet$ Moderately consistent & $97 \%$ \\
\hline & $\bullet$ Inconsistent & $0 \%$ \\
\hline$\# 3$ & Scientific resoning & $24 \%$ \\
\hline & $\bullet$ Formal reasoner & $45 \%$ \\
\hline & $\bullet$ Late transitional reasoner & $31 \%$ \\
\hline & $\bullet$ Early transitional reasoner & \\
\hline & $\bullet$ Concrete reasoner & \\
\hline
\end{tabular}

Table 1 shows characteristic of reasoning patterns which is demonstrated by students may also explain the reason for the low students' conceptual understanding score. Students do not understand well the concept of force, even after the instruction. This also becomes an indication that the instruction has not fully and explicitly taught scientific reasoning, and the learning most likely makes student memorizes problem solving algorithms by carrying out meaningless tasks or quantitative tasks only $[15,16]$.

Fig. 1 shows the student averages for reasoning pattern assessed by the Lawson test. Student demonstrated significant difficulty with identification and control of variables, correlational thinking, probabilistic thinking and hypotheticodeductive reasoning. The depiction of those reasoning patterns assessed is increasingly showing the characteristic of a concrete reasoner who uses well reasoning pattern, such as conservation (C2), while the reasoning pattern F1-F6 are either not used, or used only partially, unsystematically, and only in familiar context [13, 14]. It is important to the attention of teachers, specifically for middle and high school teacher, to identify student's scientific reasoning before instruction. In addition, identifying students' reasoning patterns is very helpful to diagnose the students who experienced more difficulty in understanding the concept, as well as a good indicator of how effective the learning that has been done in class [3].

Teachers sometimes implicitly teach scientific reasoning to students through qualitative questions and tasks, but students need to be practiced explicitly in order to become their major concern and are always accustomed in every science learning. Moreover, scientific reasoning is essential and indispensable in scientific thinking.

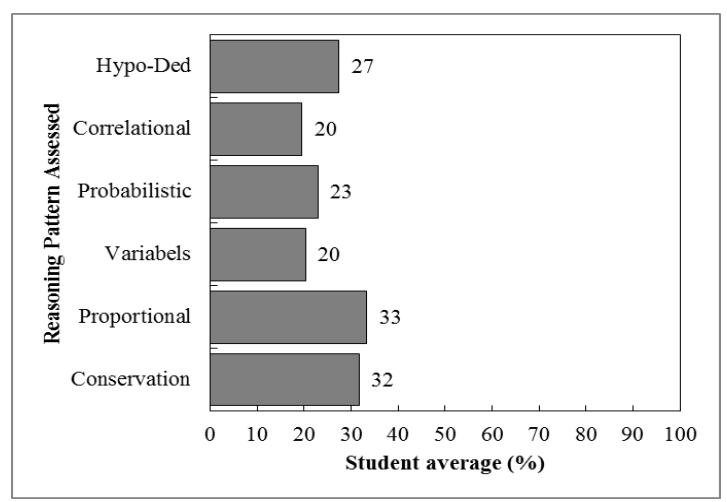

Fig. 1. Student averages on specific reasoning pattern assessed by the Lawson test

Fig. 2 shows Spearman's rank correlation between students' scientific reasoning $\left(L_{\text {post }}\right)$ and three posttest variables for 29 students: conceptual understanding $(R$ $\left.F C I_{\text {post }}\right)$, scientific consistency $\left(S C_{\text {post }}\right)$ and representational consistency $\left(R C_{\text {post }}\right)$. All correlations are statistically significant at the 0.05 level (2-tailed). The existing significant correlation between $L_{\text {post }}$ dan $R C_{\text {post }}(\rho=+0.39$ with $p=0.05$ ) has answered our first research question, as well as confirming the earlier research finding that used scientific reasoning and representational consistency prescore on the analysis $(\rho=+0.45$ with $p<0.001)$ [5].

As for our second research question is answered by the correlation coefficient between Lpost and SCpost, and between Lpost and R-FCIpost. Both are larger than those between Lpost and RCpost. Theoretically, it is in line with the explanation that in constructing a good understanding, we need scientific reasoning as an ability to develop logical relation among scientific evidence or phenomena with our prior conception by utilizing the representations [8]. This finding was also automatically supported by the depiction of student's R-FCI, RC, SC and L scores in the previous explanation. The student demonstrates moderately consistency for RC and almost all of them are inconsistent for SC, as well as they are also classified as concrete reasoner and early transitional reasoner.

Theoretically, the important characteristics of concrete reasoning is they need reference to familiar objects and descriptive properties, so that their reasoning tends to be initiated through observation $[10,13,14]$. In the context of RFCI test which was designed as isomorphic test that presents a conceptual question of the specific concept into four different representations, student who has concrete reasoning may not be familiar with the representation in this isomorphic test. In addition, concrete reasoner also demonstrates inconsistencies among the various statements they make [10, 13, 14]. This theoretical explanation is evidenced by students' consistency and reasoning scores. Therefore, these findings clearly show that there is a close relationship between consistency and scientific reasoning. 


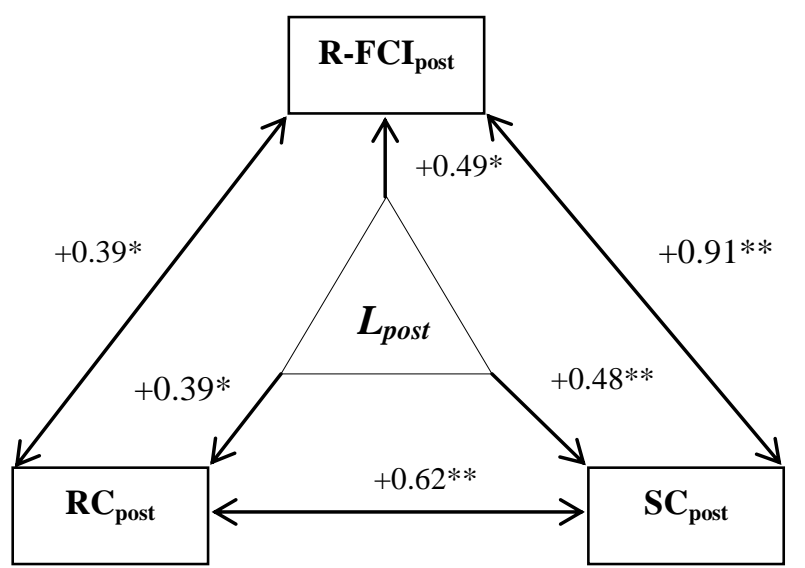

Fig. 2. Spearman's rank correlation between the Lawson posttest and three variables: conceptual understanding, scientific consistency and representational consistency. All correlations are significant at *the 0.05 level and $* *$ the 0.01 level

In Fig. 2, the relation between between RCpost and SCpost shows a strong correlation, but it does not mean a causal relationship in which a student who gets a high $\mathrm{RC}$ score will also show the high SC score. Because the ability to interpret is necessary to understand the concept, but not enough to understand the concept of physics correctly. In other words, mastering the multiple-representation does not guarantee having the correct understanding of physics concept, even though the representational ability is a prerequisite for it [7]. This explanation is also appropriate explaining the relationship between RCpost and R-FCIpost. While the relationship between the SCpost and R-FCIpost which shows a very strong correlation coefficient certainly support the notion that someone with a good understanding must be able to solve problems in different contexts and representations [6].

\section{CONCLUSION}

We have found that the correlation between student's scientific reasoning and three assessed variables: conceptual understanding of force concept, representational consistency and scientific consistency, are statistically significant. It also confirms the earlier findings of the relation between scientific reasoning and representational consistency, as well as revealing the strong correlation between scientific reasoning and scientific consistency. Despite the scientific reasoning and representational consistency influences student's performance in learning, but both have also strong correlation in which scientific reasoning is required as an underlying skill to master the consistency. Hence, it is necessary to make reasoning explicit in the instruction.

Overall, the student demonstrates either moderately consistent or inconsistency level for both consistency, while nearly half and third of the student are respectively early transitional reasoned and concrete reasoned. Student encountered the conceptual difficulty, specifically with theoretical content, which result in low conceptual understanding of the force concept and scientific consistency. Therefore, identify the students' scientific reasoning and representational consistency is required at the beginning of instruction or semester because it is useful to reveal student who has more difficulty in learning and may provide the information to develop appropriate method which overcome the pedagogical issues.

\section{Acknowledgment}

We greatly thank prospective physics teacher and faculty members for the cooperation and support in conducting this research. The author(s) received no financial support for the research, and/or authorship, except for the publication of this article from Universitas Pendidikan Indonesia.

\section{References}

[1] D.E. Meltzer, "The relationship between mathematics preparation and conceptual learning gains in physics: A possible "hidden variable" in diagnostic pretest scores," Am. J. Phys., vol. 70(12), pp. 1259-1268, December 2002.

[2] V.P. Coletta and J.A. Phillips, "Interpreting FCI scores: Normalized gain, preinstruction scores, and scientific reasoning ability," Am. J. Phys., vol. 73(12), pp. 1172-1182, December 2005.

[3] V.P. Coletta, J.A. Phillips, and J.J. Steinert, " Why you should measure your students' reasoning ability," Physc. Teach. Vol. 45, pp. 235-238, April 2007.

[4] J. Moore and L. Rubbo, "Scientific reasoning abilities of nonscience majors in physics-based courses," Phys. Rev. ST Phys. Educ. Res., vol. 8, 010106, 2012.

[5] P. Nieminen, A. Savinainen and J. Virii, "Relation between representational consistency, conceptual understanding of the force concept, dan scientific reasoning," Phys. Rev. ST. Phys. Educ. Res., vol. 8 (1), 010123 (10), 2012.

[6] D. Hestenes, "Modeling methodology for physics teachers, in the changing role of physics departments in modern universities," Proceedings of the International Conference on Undergraduate Physics Education, College Park, 1996, AIP Conference Proceedings No. 399 edited by E. Redish and J. Rigden (AIP, New York, 1997), pp. 935; Diunduh dari http:// modeling.asu.edu./r\&e/ModelingMeth-jul98.pdf

[7] P. Nieminen, A. Savinainen, and J. Virii, "Force Concept Inventorybased multiple-choice test for investigating students' representational consistency,” Phys. Rev. ST. Phys. Educ. Res., vol. 6 (2). 020109 (12), 2010 .

[8] A.E. Lawson, "Formal reasoning ability and misconceptions concerning genetics and natural selection," J. Res. Sci. Teach., vol. 25(9), pp. 733746, 1988.

[9] A.E. Lawson, “ The nature and development of scientific reasoning: A synthetic view,” Int. J. Sci. Math. Educ., vol. 2, pp. 307-338, 2004.

[10] A.E. Lawson, "Science teaching and development of thinking," United Stated of America: Wadsworth, 1995.

[11] J.W. Renner and A.E. Lawson, "Promoting intellectual development through science teaching," Phys. Teach., vol. 11, pp. 273-276, 1973.

[12] A.E. Lawson, "The development and validation of classroom test of formal reasoning," J. Res. Sci. Teach., vol. 15(1), pp. 11-24, 1978.

[13] A.E. Lawson, "Teaching inquiry science in middle and secondary schools," Sage, Publications, Inc: Thousand Oaks, CA, 2010.

[14] R.G. Fuller, R. Karplus and A.E. Lawson, "Can physics develop reasoning?," Phys. Today, vol. 30(2), pp. 23-28, 1977.

[15] D. Hestenes, M. Wells, and G. Swackhamer, "Force Concept Inventory," Phys. Teach., vol 30, 141-158, March 1992. The 1995 revision by Halloun, Hake, Mosca, \& Hestenes, which was used in this study, is available online [password protected] at http://modeling.asu.edu/ R\&E/Research.html

[16] N. Lasry, N. Finkelstein and E. Mazur, "Are most people too dumb for physics?," Phys. Teach., vol. 47, pp. 418-422, 2009. 